\title{
Stimulus specificity in the acquisition and extinction of conditioned taste aversion
}

\author{
EDGAR H VOGEL ${ }^{1}$, FABIÁN A SOTO ${ }^{1}$, MARÍA E CASTRO ${ }^{2}$ and PAOLA A SOLAR ${ }^{2}$
}

${ }^{1}$ Universidad de Talca, Talca, Chile.

${ }^{2}$ Universidad del Mar, Viña del Mar, Chile.

\begin{abstract}
An experiment evaluated whether the acquisition and extinction of conditioned taste aversion in the rat is stimulus-specific by testing the degree of response transfer between sweet and salty tastes. Animals in the paired-same and paired-different groups received a presentation of a gustatory CS and a cyclophosphamide injection US. Nonconditioned control groups received unpaired CS /US presentations or the CS followed by a vehicle injection. Taste avoidance was evaluated in three nonreinforced test sessions. In the paired-same, unpaired and vehicle groups, all test sessions were conducted with the same flavor as originally used in training, whereas the paired-different group was tested with a novel flavor on the first and second sessions and with the originally trained flavor in last session. Stimulus specific acquisition was apparent in the first test session, when the animals in the group paired-same exhibited lower fluid intake than the other three groups. Evidence of specificity of extinction was apparent in the last test session, when animals in the group paired-different exhibited lower fluid intake than the other three groups. These results provide further evidence of stimulus specificity in acquisition and extinction of conditioned taste aversion, supporting the associative interpretation of these phenomena.
\end{abstract}

Key terms: conditioning, cyclophosphamide, extinction, generalization, stimulus specificity, taste aversion.

\section{INTRODUCTION}

The present research evaluated stimulus specificity of acquisition and extinction of conditioned taste aversion. Conditioned taste aversion results from the exposure of an animal to a particular taste followed by gastrointestinal malaise, typically induced by drugs $(6,9)$ or radiation (5). After a single CS/US pairing, the animal avoids further consumption of the relevant flavor, that is, it acquires a conditioned taste aversion (CTA). The conditioned response diminishes if the animal is repeatedly exposed to the relevant taste not followed by illness, called extinction of CTA.

The usual explanation of CTA is associative. That is, it is assumed that acquisition and extinction of CTA result from the strengthening and weakening of CS-US associations, respectively. One important prediction from this associative account is that changes in behavior must be in some degree specific to the particular taste presented during acquisition and extinction of CTA, and should not affect responses to a large range of tastes.

Stimulus specificity is strongly tied to stimulus generalization. Both can be viewed as opposite aspects of the transfer of responses from one stimulus to another on the basis of their similarity. Indeed, an associative account of CTA predicts that the "generalization decrement" (i.e., degree of specificity) is a function of the similarity between trained and tested flavors. This prediction is supported by the empirical evidence. For instance, several studies have demonstrated a generalization decrement as a function of increasing and decreasing the concentration of the originally trained solution (11, 13-14). Other studies have 
encountered generalization functions for chemically different, but qualitatively similar tastes, such as salty $(4,9-10)$ or sweet (14). Finally, studies that have included tests of generalization between qualitatively different tastes have shown little or no generalization of CTA, which is the most stringent evidence of specificity $(1-2,4,7-8,12)$.

In contrast to the relatively convincing evidence of specificity of acquisition of CTA, there is not much information on extinction. For instance, Nachman (9) conducted an extinction experiment using the flavor of $\mathrm{LiCl}$ dissolved in water as the CS, and its poisoning effects as the US. After conditioning, the animals received several "extinction trials" with an innocuous, differently flavored solution. The results indicate that after extinction, when the animals were tested again with the originally trained CS, the degree of CTA was a function of the initial level of generalization between the taste of $\mathrm{LiCl}$ and the extinguished flavor. That is, stimuli with high generalized taste aversion promoted high levels of generalization of extinction to the trained CS, whereas stimuli that showed less generalized taste aversion promoted less generalization of extinction to the trained CS.

Unfortunately, Nachman's (9) study presents several problems. First, it was not possible to evaluate taste avoidance to the originally trained CS after its extinction ( $\mathrm{LiCl}$ could not be consumed without its gastrointestinal consequences), and compare it against avoidance to nondirectly extinguished stimuli. Second, there was no control for the general changes in responsiveness that may be produced by the repeated presentation of a neutral stimulus during the extinction procedure. Finally, the study did not control the effect of unpaired presentations of the CS and the US.

The present experiment evaluated stimulus specificity of acquisition and extinction of CTA avoiding the listed problems in previous research. To achieve this, two groups of rats received presentations of a taste followed by cyclophosphamide-induced illness, and then were tested with repeated presentations of a taste in an extinction procedure (see Table 1). For group paired-same, all tests were conducted with the same taste as the one used during training, whereas for group paired-different, the first two tests used a different taste, and the final test was conducted with the originally-trained taste. On test 1 , the comparison of these groups evaluated stimulus specificity of acquisition, and on test 3 the comparison evaluated stimulus specificity of extinction. Two control groups were used. Group unpaired was treated exactly like group paired same, but there was a 24-hr interval between the flavor and the drug. This group provided a control for the unconditioned effects of cyclophosphamide upon subsequent preference or rejection of tastes and for the effects of pairing illness with the drinking situation. Group vehicle received a taste paired with injection of a saline solution and was always tested with the same taste presented during training. This group provided a control for the effects of pairing the taste with the injection and for the repeated presentation of a neutral taste during extinction. Since the main objective was to evaluate stimulus specificity instead of stimulus generalization, the response transfer was evaluated through two qualitatively different stimuli ( $\mathrm{NaCl}$ and Saccharin).

\section{METHOD}

\section{Animals and apparatus}

Thirty-two male albino rats (purchased from Pontificia Universidad Católica de Chile breeding colony, Santiago, Chile) weighing 400-450 g., were housed in individual cages, with free access to food. Mounted on each cage was a holder which could support a $100-\mathrm{ml}$ plastic syringe adapted with a glass drinking spout in the bottom. All fluids were delivered in these syringes. All experimental treatments took place in the individual home cages. The rats were adapted, over a 7-day period, to 15 minutes of access to water per day. After adaptation, the rats were matched into four groups based on their water intake $(n=8)$. 
TABLE 1

Experimental design. A and B represent the presentation of different flavors dissolved in water, which could be followed by a cyclophosphamide (CY) or a physiological saline

(Sal) injection after a 30-minutes delay (-) or a 24-hours delay (/).

\begin{tabular}{|c|c|c|c|c|}
\hline & \multirow{2}{*}{$\frac{\text { Acquisition }}{\text { First Exposure }}$} & \multicolumn{3}{|c|}{ Extinction } \\
\hline & & Test 1 & Test 2 & Test 3 \\
\hline Paired-Same & A - CY & A & A & A \\
\hline Paired-Different & $\mathrm{A}-\mathrm{CY}$ & B & B & A \\
\hline Unpaired & $\mathrm{A} / \mathrm{CY}$ & A & A & A \\
\hline Vehicle & A - Sal & A & A & A \\
\hline
\end{tabular}

\section{Procedure}

On the day following the last day of adaptation (day 1), animals in the group unpaired were exposed to a novel taste, by replacing the plain water with flavored water during the 15-min drinking period. On this day, all other groups received the regular 15-min access to plain water.

The next day (day 2) was the conditioning day. Animals in the group unpaired received plain water, and all other groups received flavored water. For half of the animals in each group, the flavored water consisted of a $0.05 \% \mathrm{NaCl}$ solution, and for the other half, a $0.1 \%$ saccharin solution. Thirty minutes after the drinking period, animals in groups paired-same, paired-different and unpaired received an intraperitoneal injection of cyclophosphamide $(50 \mathrm{mg} / \mathrm{Kg})$. Animals in the vehicle group were injected with an equivalent volume of physiological saline.

During the next two days all animals returned to the regular $15-\mathrm{min}$ access to plain water. On day 5 the first test was conducted, which consisted of the replacement of plain water with flavored water. For the animals in groups pairedsame, unpaired and saline the flavor was the same as that used in the first exposure to flavored water (hereafter designated as "flavor A"), whereas for animals in the group paired-different the test stimulus was a different novel flavor (i.e., $\mathrm{NaCl}$ for subjects originally trained with saccharin, and vice versa; hereafter designated as "flavor B").

Two additional testing sessions were conducted on days 15 and 18 . The second testing session was identical to the first test, that is, groups paired-same, unpaired, and vehicle received flavor $A$, and the group paired-different received flavor B. On the third testing session all animals were exposed to flavor A.

In summary, groups paired-same, unpaired and vehicle were trained and tested always with the same flavor (flavor A). For the group paired-different, the first two tests were conducted with a flavor different from that used in training (i.e., training with flavor $\mathrm{A}$ and testing with flavor B) and the third test used the same flavor originally paired with the US (flavor A).

\section{Scoring and data analysis}

CTA was assessed through a preference ratio, calculated for each animal by dividing their water intake during the corresponding test by the mean water intake on the two immediately preceding days. A preference ratio of less than 1 suggests that an aversion was developed to the taste, whereas a value equal or higher than 1 indicates the absence of aversion. 
A 2 (Stimulus quality: $\mathrm{NaCl}$, saccharin) x 4 (Group: paired-same, paired-different, unpaired, vehicle) x 4 (session: first exposure, test 1 , test 2 , test 3 ) mixed-design ANOVA with Preference Ratio as the dependent variable was used for statistical interpretation. When appropriate, a Fisher PLSD post hoc test was performed to assess differences between pairs of groups and between pairs of sessions. All statistical differences were considered significant at the 0.05 level.

\section{RESULTS}

One animal in group unpaired died during the experiment and its data were discarded from the analyses.

The $2 \times 4 \times 4$ mixed-design ANOVA indicated reliable main effects of stimulus quality $(\mathrm{F}(1,23)=21.204, \mathrm{p}<0.001)$, group $(\mathrm{F}(3,23)=20.352, \mathrm{p}<0.001)$, and session
$(\mathrm{F}(3,69)=7.52, \mathrm{p}<0.001)$, and reliable interactions between group and session ( $\mathrm{F}$ $(9,69)=12.927, p<0.001)$ and between stimulus quality, group and session ( $F$ $(9,69)=3.408, \mathrm{p}<0.002)$. To understand the meaning of these effects three types of statistical analyses were performed. First, differences between groups within each session were examined by ANOVAs testing the simple main effects of group within each session, followed, where appropriate, by pairwise post hoc tests. Second, within group differences over the sessions were evaluated by pairwise dependent post hoc tests. Third, to evaluate the generality of the findings regarding specificity of acquisition and extinction of CTA, separate descriptive analyses for the two stimulus qualities were conducted. The findings of major interest are described in Figure 1, which depicts the group's preference ratios over the sessions, and can be described as follows:

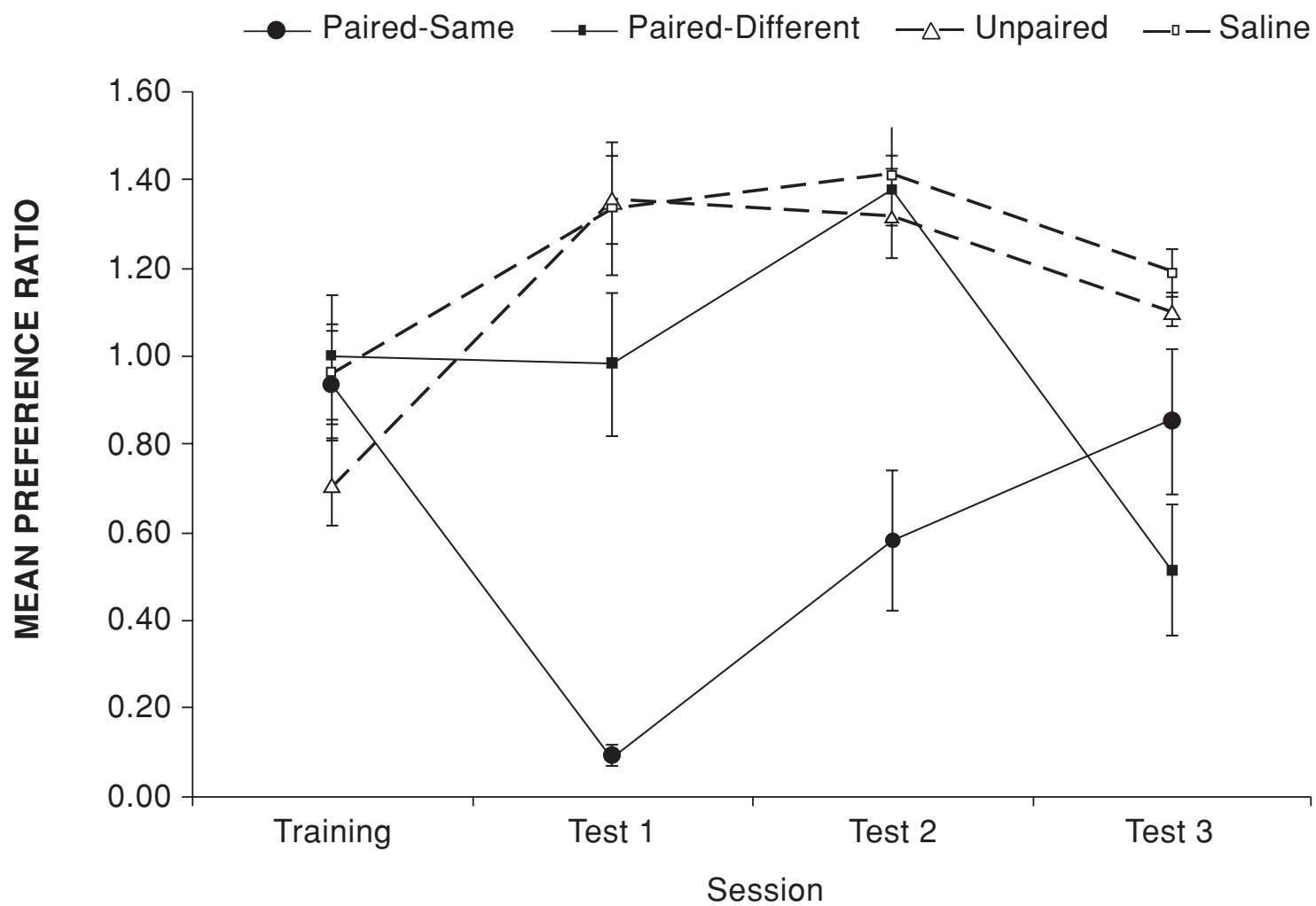

Figure 1: Mean preference ratios for all groups in the experiment calculated for training session (baseline) and testing session. The error bars represent the standard error of the means. 
1. Training session. As can be seen in Figure 1, there were no major differences among the groups in the first exposure to flavor A. This result was confirmed by an ANOVA testing the simple main effect of group in the training session (F $(3,23)$ $=1.808, \mathrm{p}>0.1)$. Consistent with previous findings reporting "neophobia" (i.e., a transient tendency to avoid a novel flavor; [3]), most animals presented preference ratios slightly below 1 in this first exposure to flavor A.

\section{Testing session 1. Group differences arose} during the first testing day $(F \quad(3,23)$ $=30.998, \mathrm{p}<0.001)$. As can be seen in Figure 1 , group paired-same showed the lowest preference ratios and an almost complete suppression of flavor A intake. As expected, groups unpaired and vehicle presented the highest preference ratios for flavor $\mathrm{A}$, while group paired-different reached an intermediate level of fluid intake. The pairwise comparisons confirm the reliability of these observations. First, group pairedsame presented a preference ratio significantly lower than groups unpaired and vehicle $(\mathrm{ps}<0.001)$ and a significant decrement from the training session to the testing session $1(\mathrm{p}<0.001)$, which is an indication of conditioned taste aversion. Second, group paired-different presented a preference ratio significantly higher than group paired-same $(\mathrm{p}<0.001)$ and no reliable difference between training and testing session $1(p>0.9)$, which supports the hypothesis that CTA is stimulus specific. Additionally, group paired-different also differed from groups unpaired and vehicle ( $\mathrm{ps}<0.031$ ), which may be explained by the fact that in testing session 1 , the different group was exposed again to a novel flavor B (neophobia), whereas the unpaired and vehicle groups received the already familiar flavor A. This last observation is supported by the fact that both groups (unpaired and vehicle) presented a reliable increase in preference ratio from training to test 1 ( $\mathrm{p}<$ 0.006 ) and in the very similar preference ratios exhibited by group paired-different in the first exposure to flavor A (training session) and the first exposure to flavor B (testing session 1).
3. Testing session 2. Reliable differences between groups were found again during testing session $2(\mathrm{~F}(3,23)=12.894$, $\mathrm{p}<0.001$ ), with post hoc tests indicating that group paired-same reliably differed from the other three groups (ps <0.001), which, in turn, did not differ from each other ( $\mathrm{ps}$ $>0.05)$. The fact that animals in group paired-same retain some degree aversion in this second test, which is not present at all in group paired-different, provides further evidence of specificity of CTA. Additionally, in this second test group paired-same exhibited an increase in its mean preference ratio as compared to testing 1 ( $\mathrm{p}<0.003)$, probably due to extinction caused by previous exposure to the CS not followed by illness. Interestingly, group paired-different also showed a reliable increase in responding from test 1 to test 2 ( $p$ $<0.05$ ), which is consistent with the increase observed in the unpaired and vehicle groups between training and test 1 (a "release" from neophobia after two presentations of the same flavor).

\section{Testing session 3. The ANOVA indicates} reliable differences among the groups ( $\mathrm{F}(3$, 23) $=13.677, p<0.001)$. As can be seen in Figure 1, group paired-same showed a mean preference ratio that was even higher than that observed during the preceding test $(\mathrm{p}<$ $0.001)$ but still lower than nonconditioned groups ( $\mathrm{ps}<0.043$ ), evidencing an ongoing process of extinction. The more interesting results in this test are from group paireddifferent, which was tested for the first time with the taste originally paired with illness during training. This group, which showed no indication of flavor aversion during the first two tests, now showed a clear decrement in the intake of flavor A. Post hoc tests indicated that the preference ratio of group paired-different was significantly lower than that of the vehicle and unpaired groups (ps <0.001) and, most importantly, lower than that of the almost-extinguished paired-same group $(\mathrm{p}<0.007)$. The existence of robust CTA in the paired-different group is also supported by the between sessions comparisons, since its mean preference ratio in test 4 was significantly lower than that in every other test ( $\mathrm{ps}<0.002)$. 
To summarize, there was a reliably greater conditioned response in group paired-same than in group paired-different in tests 1 and 2 , which provides evidence for specificity of acquisition, and a reliably greater conditioned response in group paired-different than in group paired-same in test 3, which provides evidence for specificity of extinction. The reliability of these findings was confirmed through the between sessions analysis. The results from control groups suggest that acquisition and extinction of CTA cannot be explained in terms of the mere exposure to the CS and US, uncorrelated or in isolation.

In addition to the effects reported above, the global mixed ANOVA previously introduced revealed a reliable main effect of stimulus quality and a reliable stimulus quality $\mathrm{x}$ session $\mathrm{x}$ group interaction. These anomalous effects do not affect the conclusion of the experiment, since they can be understood as the rats' preference for $\mathrm{NaCl}$ over saccharine over the entire experiment. This can be seen in the fact that the mean preference ratio of the two nonconditioned groups trained and tested with saccharin was 0.99 , which indicates that rats consumed as much saccharin solution as plain water. In contrast, the mean preference ratio for these groups trained and tested with $\mathrm{NaCl}$ was 1.31 , indicating that they drank more $\mathrm{NaCl}$ than water. The difference in these mean preference ratios was found reliable (Mean $\mathrm{D}=-0.4033, \mathrm{p}<0.001$ ).

Likewise, the stimulus quality $\mathrm{x}$ session $\mathrm{x}$ group interaction can be largely explained by the rats' preferences for $\mathrm{NaCl}$ over saccharine. These different patterns of results do not affect the critical comparisons used to evaluate stimulus specificity. This can be seen in Table 2, which presents the data separately for the two tastes used in training. As can be seen in the table, the general pattern of results over sessions for animals in group paired same is very similar for animals trained with saccharine and $\mathrm{NaCl}$, with a decrease in test 1 (aversion) followed by a progressive increase in test 2 and test 3 (extinction). Likewise, regardless of the trained taste, animals in the paired-different group presented a lower preference ratio than that of the paired same-group in test 1 (specificity of acquisition), and a higher preference ratio than that of the paired same-group in test 3 and decrement from training to test 3 (specificity of extinction).

TABLE 2

Mean preference ratios (+/- standard error of the mean) for groups paired same, paireddifferent, unpaired and vehicle over sessions. The data are disaggregated by the taste used in the training session (saccharine or saline).

\begin{tabular}{|c|c|c|c|c|}
\hline & \multicolumn{4}{|c|}{ Saccharin } \\
\hline & $\begin{array}{c}\text { Training } \\
\text { Mean (SEM) }\end{array}$ & $\begin{array}{c}\text { Test } 1 \\
\text { Mean (SEM) }\end{array}$ & $\begin{array}{c}\text { Test } 2 \\
\text { Mean (SEM) }\end{array}$ & $\begin{array}{c}\text { Test } 3 \\
\text { Mean (SEM) }\end{array}$ \\
\hline Paired-Same & $0,70(0,18)$ & $0,08(0,02)$ & $0,56(0,34)$ & $0,56(0,26)$ \\
\hline Paired-Different & $0,69(0,10)$ & $1,05(0,33)$ & $1,51(0,04)$ & $0,15(0,03)$ \\
\hline Unpaired & $0,59(0,17)$ & $1,19(0,08)$ & $1,17(0,18)$ & $1,13(0,07)$ \\
\hline \multirow[t]{3}{*}{ Vehicle } & $0,79(0,16)$ & $0,98(0,08)$ & $1,18(0,13)$ & $1,09(0,08)$ \\
\hline & \multicolumn{4}{|c|}{$\mathrm{NaCl}$} \\
\hline & $\begin{array}{c}\text { Training } \\
\text { Mean (SEM) }\end{array}$ & $\begin{array}{c}\text { Test } 1 \\
\text { Mean (SEM) }\end{array}$ & $\begin{array}{c}\text { Test } 2 \\
\text { Mean (SEM) }\end{array}$ & $\begin{array}{c}\text { Test } 3 \\
\text { Mean (SEM) }\end{array}$ \\
\hline Paired-Same & $1,16(0,06)$ & $0,10(0,05)$ & $0,60(0,08)$ & $1,14(0,07)$ \\
\hline Paired-Different & $1,30(0,15)$ & $0,91(0,09)$ & $1,23(0,12)$ & $0,87(0,13)$ \\
\hline Unpaired & $0,81(0,11)$ & $1,47(0,14)$ & $1,43(0,10)$ & $1,08(0,05)$ \\
\hline Vehicle & $1,12(0,13)$ & $1,69(0,12)$ & $1,64(0,06)$ & $1,28(0,03)$ \\
\hline
\end{tabular}




\section{DISCUSSION}

The present experiment aimed to evaluate stimulus specificity in the acquisition and extinction of conditioned taste aversion. Stimulus specificity in the acquisition of conditioned taste aversion was demonstrated by the strong suppression of taste consumption exhibited by rats tested with a stimulus previously paired with illness (test 1 for group paired-same), a result that was not observed in rats tested with a different stimulus from that used as CS (test 1 for group paired-different). These results are consistent with the usual explanation of acquisition of CTA based on the strengthening of Pavlovian associations between the relevant stimulus and the drug.

Stimulus specificity of extinction of CTA was demonstrated since it occurred only when the procedure involved repeated presentations of the originally trained CS. It was shown that when the extinction procedure involved presentations of the relevant CS, the level of taste consumption was highly recovered (test 3 for group paired-same), and more important, to a higher level than the consumption showed when another taste was extinguished (test 3 for group paired-different). In the last case, rats showed avoidance of taste when tested with a stimulus previously paired with illness, even after the exposure to an extinction procedure that involved repeated presentations of another taste. Again, these results are consistent with an explanation of extinction as the weakening of the original Pavlovian taste-illness association.

Therefore, these results are well suited to an explanation of acquisition and extinction of learned taste aversions in terms of the gain and loss (or interference) of associations between illness and the specific taste paired with it. This confirms the results of previous studies on generalization of CTA $(4,8-12)$, and, to our knowledge, provides the first clear evidence of specificity in the extinction of CTA, overcoming some of the methodological problems of previous studies (9) and demonstrating stimulus specificity (and not stimulus generalization) by the use of qualitatively different stimuli during training and test.

\section{ACKNOWLEDGEMENTS}

This research was supported by Fondecyt Grant No. 1040109 to E. H. Vogel.

\section{REFERENCES}

1. CHOTRO MG, ALONSO G (1999) Effects of stimulus preexposure on the generalization of conditioned taste aversions in infant rats. Dev Psychobiol 35: 304-317

2. CHOTRO MG, AlONSO G (2003) Stimulus preexposure reduces generalization of conditioned taste aversions between alcohol and non-alcohol flavors in infant rats. Behav Neurosci 117: 113-122

3. DOMJAN M (1977) Selective suppression of drinking during a limited period following aversive drug treatment in rats. J Exp Psychol Anim Behav Process 3: 66-76

4. FORMAKER BK, HILL DL (1990) Alteration of salt taste perception in the developing rat. Behav Neurosci 104: 356-364

5. GARCÍA J, KIMELDORF DJ, KOELLING RA (1955) Conditioned aversion to saccharin resulting from exposure to gamma radiation. Science 122: 157-158

6. HAYES UL, CHAMBERS KC (2005) High doses of vasopressin delay the onset of extinction and strengthen acquisition of LiCl-induced conditioned taste avoidance. Physiol Behav 84:625-633

7. KIEFER SW, MAHADEVAN, RS (1993) The taste of alcohol for rats as revealed by aversion generalization tests. Chem Senses 18: 509-522

8. MACKINTOSH NJ, KAYE H, BENNETT CH (1991) Perceptual learning in flavor aversion conditioning. Q J Exp Psychol 43B: 297-322

9. NACHMAN M (1963) Learned aversion to the taste of lithium chloride and generalization to other salts. J Comp Physiol Psychol 56: 343-349

10. NACHMAN M, RAUSCHENBERGER J, ASHE JH (1977) Stimulus characteristics in food aversion learning. In: MILGRAM NW, KRAMES L, ALLOWAY TM (eds) Food Aversion Learning. New York: Plenum Press. Pp: 105-131

11. NOWLIS GH (1974) Conditioned stimulus intensity and acquired alimentary aversions in the rat. J Comp Physiol Psychol 86: 1173-1184

12. NOWLIS GH, FRANK ME, PFAFFMAN C (1980) Specificity of acquired aversions to taste qualities in hamsters and rats. J Comp Physiol Psychol 94: 932-942

13. SMITH DV, THEODORE RM (1984) Conditioned taste aversions: Generalization to taste mixtures. Physiol Behav 32: 983-989

14. TAPPER DN, HALPERN BP (1968) Taste: A behavioral categorization. Science 161: 708-710 
